\title{
The DbpA catalytic core unwinds double-helix substrates by directly loading on them
}

\author{
JARED J. CHILDS, RILEY C. GENTRY, ANTHONY F.T. MOORE, and EDA KOCULI \\ Department of Chemistry, University of Central Florida, Orlando, Florida 32816, USA
}

\begin{abstract}
DbpA is a DEAD-box RNA helicase implicated in the assembly of the large ribosomal subunit. Similar to all the members of the DEAD-box family, the DbpA protein has two N-terminal RecA-like domains, which perform the RNA unwinding. However, unlike other members of this family, the DbpA protein also possesses a structured C-terminal RNA-binding domain that mediates specific tethering of DbpA to hairpin 92 of the Escherichia coli 235 ribosomal RNA. Previous studies using model RNA molecules containing hairpin 92 show that the RNA molecules support the DbpA protein's double-helix unwinding activity, provided that the double helix has a $3^{\prime}$ single-stranded region. The $3^{\prime}$ single-stranded region was suggested to be the start site of the DbpA protein's catalytic unwinding activity. The data presented here demonstrate that the single-stranded region $3^{\prime}$ of the doublehelix substrate is not required for the DbpA protein's unwinding activity and the DbpA protein unwinds the double-helix substrates by directly loading on them.
\end{abstract}

Keywords: DbpA; DEAD-box protein; ribosome maturation factor; RNA helicase; ribosome assembly

\section{INTRODUCTION}

DEAD-box proteins are a family of enzymes that use the energy of ATP binding and hydrolysis to facilitate RNA conformational changes in many pathways of RNA metabolism, including RNA splicing, ribosome maturation, transcription, RNA transport, and RNA degradation (Tanner and Linder 2001; Cordin et al. 2006; Pyle 2008; Pan and Russell 2010; Linder and Jankowsky 2011). DEAD-box proteins are part of the helicase 2 superfamily of enzymes (Caruthers and McKay 2002) and, in vitro, most, but not all, show nonprocessive RNA helicase activity on model substrates (Cordin et al. 2006; Linder and Jankowsky 2011). However, unlike the DNA and viral RNA helicases of the SF2 family, which start their actions at the single-stranded region of the helical substrate and translocate toward the opposite end, DEADbox proteins directly load on the double-helix substrate and unwind it without translocation (Pyle 2008; FairmanWilliams et al. 2010; Pan and Russell 2010).

The DEAD-box proteins' direct loading mechanism was originally proposed based on the crystal structure of the Vasa protein in complex with a single-stranded RNA construct and the nonhydrolyzable ATP analog, AMP-PNP (Sengoku et al. 2006). This structure showed that the single-stranded RNA bound to the catalytic core of the Vasa protein had a kinked structure that would be incompatible with

Corresponding author: eda.koculi@ucf.edu

Article published online ahead of print. Article and publication date are at http://www.rnajournal.org/cgi/doi/10.1261/rna.052928.115. the conformation of that strand on an RNA double helix. Hence, it was suggested that DEAD-box helicases unwind their substrates by loading on one strand of the double helix and kinking it, which forces the release of the second RNA strand. Other crystal structures of DEAD-box proteins in complex with a single-stranded RNA and nonhydrolyzable ATP analogs agreed with the data from the Vasa structure (Andersen et al. 2006; Bono et al. 2006; Del Campo and Lambowitz 2009). In addition, an extensive series of kinetic experiments using purified Saccharomyces cerevisiae proteins Mss116p and Ded1p and Neurospora crassa protein CYT-19 demonstrated that the role of the single-stranded RNA extensions is for tethering of the above DEAD-box proteins to the RNA molecule rather than for initiation of the unwinding process (Yang et al. 2007).

This paper addresses the mechanism of action of E. coli DbpA, a DEAD-box protein involved in assembly of the large ribosomal subunit (Fuller-Pace et al. 1993; Diges and Uhlenbeck 2001; Sharpe Elles et al. 2009), using a series of model helicase substrates. DbpA resembles other DEADbox proteins in having two RecA-like catalytic domains that bind RNA and hydrolyze ATP, but it is unusual in having a structured C-terminal domain that binds tightly and

(C) 2016 Childs et al. This article is distributed exclusively by the RNA Society for the first 12 months after the full-issue publication date (see http://rnajournal.cshlp.org/site/misc/terms.xhtml). After 12 months, it is available under a Creative Commons License (Attribution-NonCommercial 4.0 International), as described at http://creativecommons.org/licenses/ by-nc/4.0/. 
specifically to helix and hairpin 92 of the $23 \mathrm{~S}$ ribosomal RNA (Kossen and Uhlenbeck 1999; Diges and Uhlenbeck 2001; Tsu et al. 2001; Karginov et al. 2005; Wang et al. 2006; Hardin et al. 2010). Model DbpA substrates containing helix and hairpin 92 and short RNA helices positioned $3^{\prime}$ or $5^{\prime}$ of helix 92 supported DbpA catalytic activity, provided that the double-helix substrates possess a 4-nucleotide (nt)-long $3^{\prime}$ single-stranded region (Diges and Uhlenbeck 2001, 2005). In analogy to the single-stranded extensions required by other DNA and viral RNA helicases of the SF2 family, it was proposed that the single-stranded region acted as the entry site for the DbpA protein's catalytic domains into the double-helix substrates (Diges and Uhlenbeck 2005).

A recent crystal structure of the C-terminal domain of YxiN, a Bacillus subtilis ortholog of DbpA, bound to helices 90, 91, and 92 of the $23 \mathrm{~S}$ ribosomal RNA (rRNA), shows extensive specific interactions between the protein and helix and hairpin 92, as predicted from biochemical data and nonspecific electrostatic interactions between the protein's lysine and arginine residues and the phosphate backbone of helix 90 (Hardin et al. 2010). Model studies suggest that in the absence of helix 90 the C-terminal of YxiN interacts with the phosphate groups on the single-stranded region $5^{\prime}$ or $3^{\prime}$ of helix 92. These single-stranded regions are believed to be required for tight binding of the YxiN C-terminal domain to RNA (Tsu et al. 2001; Wang et al. 2006). Hence, the YxiN C-terminal domain uses different modes of interaction with different RNA constructs. More importantly, the interaction of the DEAD-box proteins' catalytic core with the phosphate backbone of one of the strands of the double-helix substrate is required for DEAD-box proteins' helicase activity (Linder and Jankowsky 2011; Henn et al. 2012; Putnam and Jankowsky 2013) suggesting the interaction of the YxiN/ DbpA C-terminal domain with an RNA molecule could dictate both which regions of that RNA are accessible for the YxiN/DbpA core to act upon and the stability of the RNA-protein complex. Thus, it is possible that the previous observation for an apparent requirement of a single-stranded region $3^{\prime}$ of the double-helix substrate to support the helicase activity of DbpA could be a consequence of different modes of interaction of the DbpA C-terminal domain with different model RNA molecules. In an attempt to examine this possibility further, this paper focuses on the role of the singlestranded residues $3^{\prime}$ of the double-helix substrate on the DbpA protein's catalytic activity.

\section{RESULTS}

\section{2'-OH moieties required for the catalytic activity of DbpA}

To investigate where DbpA starts its unwinding action, we took advantage of the fact that DbpA, like most DEAD-box helicases, is an RNA helicase and does not unwind doublestranded DNA helices (Diges and Uhlenbeck 2001).
Moreover, previous studies have used model substrates that lacked RNA 2'-OH groups to investigate Dedlp and Mss116p helicase action initiation sites (Yang et al. 2007).

The molecules initially used for this study are shown in Figure 1A. Molecule A:X consists of a 32-nt-long RNA containing helix 92 and a 15-residue single-stranded region $5^{\prime}$ of helix 92 annealed to a 9-nt-long RNA strand. This model molecule has been used extensively to investigate DbpA's and YxiN's functional properties (Diges and Uhlenbeck 2001, 2005; Elles and Uhlenbeck 2008; Theissen et al. 2008; Aregger and Klostermeier 2009; Henn et al. 2012). Molecule B:X consists of a 32-nt-long DNA-RNA chimera, in which 15 RNA residues $5^{\prime}$ of helix 92 have been substituted by DNA residues, annealed to the 9-nt-long RNA. In the molecule A:Y, the 32-nt-long RNA construct is annealed to a 9-nt-long DNA construct.

The ability of the constructs to support the helicase activity of DbpA was investigated by native gel electrophoresis as previously described (Diges and Uhlenbeck 2001, 2005; Elles and Uhlenbeck 2008). Figure 1B shows the dependence of the fraction unwound versus the reaction time. The molecule B:X, which contains 15 bases $5^{\prime}$ of hairpin 92 changed to DNA, is unable to support the helicase activity of DbpA, while the molecule $\mathrm{A}: \mathrm{Y}$, in which the short 9-nt-long top strand is changed to DNA, supports the helicase activity of DbpA. The higher observed rate constant of unwinding in the presence of molecule A:Y when compared to molecule $\mathrm{A}: \mathrm{X}$ is a consequence of the fact that DNA-RNA helices are less stable than RNA-RNA helices, and the helix stability has been shown to influence both the observed rate constant and the extent of the DEAD-box proteins' unwinding activity (Chen et al. 2008). Thus, the presence of $2^{\prime}-\mathrm{OH}$ on one strand of the double-helix substrate is sufficient for a helix to support DbpA helicase activity. This result agrees with data from other DEAD-box proteins (Yang et al. 2007). In addition, these experiments imply that the top stand of the double-helix is mechanistically unimportant for the DbpA protein helicase activity.

Previous studies performed with A:X and other small model molecules show that DbpA protein is unable to unwind $100 \%$ of its substrates (Diges and Uhlenbeck 2001, 2005; Elles and Uhlenbeck 2008; Henn et al. 2008). This is believed to be a consequence of incorrectly formed alternative RNA structures and helix annealing. Spontaneous reannealing is another process that could affect the extent of double-helix unwinding by DbpA. At $1 \mathrm{nM}$ RNA molecule, a small amount of RNA spontaneously reanneals; however, the observed rate constant of this process is slow and it does not contribute significantly to the observed extent of unwinding within the 30 min duration of our experiments. Our observed rate constant of spontaneous annealing in the presence of $1 \mathrm{nM}$ construct $\mathrm{A}$ and $\mathrm{X}$ is $0.006 \pm 0.003$ $\mathrm{min}^{-1}$, which is consistent with previously performed experiments with constructs $\mathrm{A}$ and $\mathrm{X}$ and at the same construct concentration (Diges and Uhlenbeck 2001). 
A

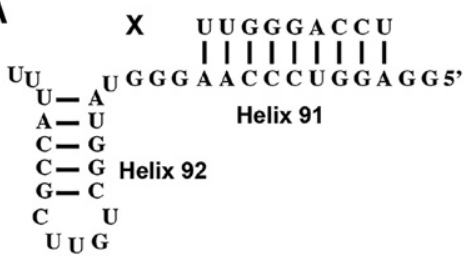

A

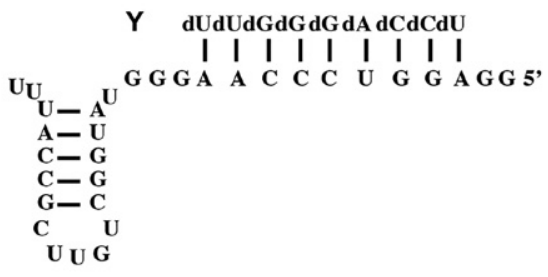

A

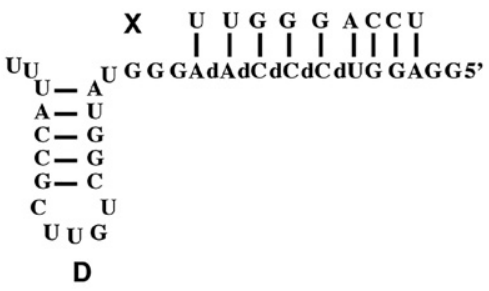

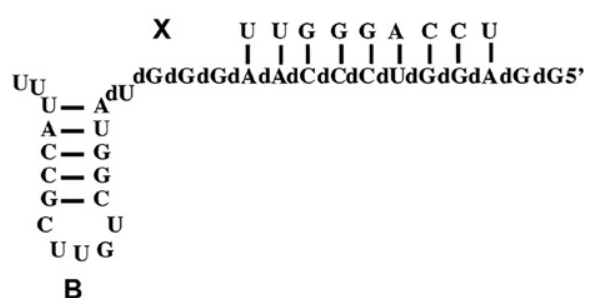
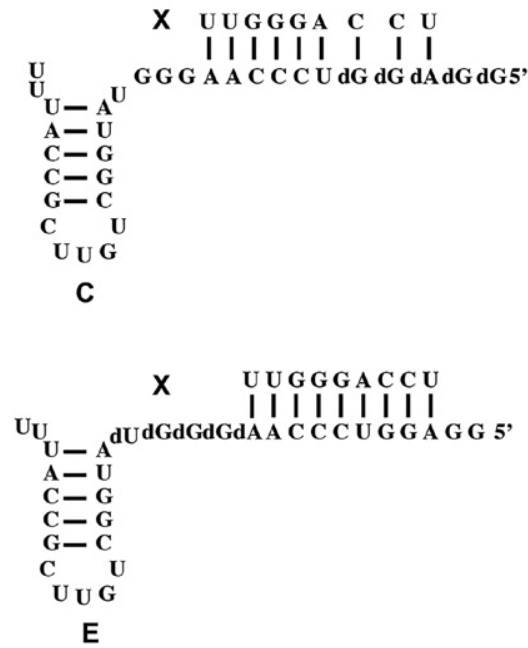

B

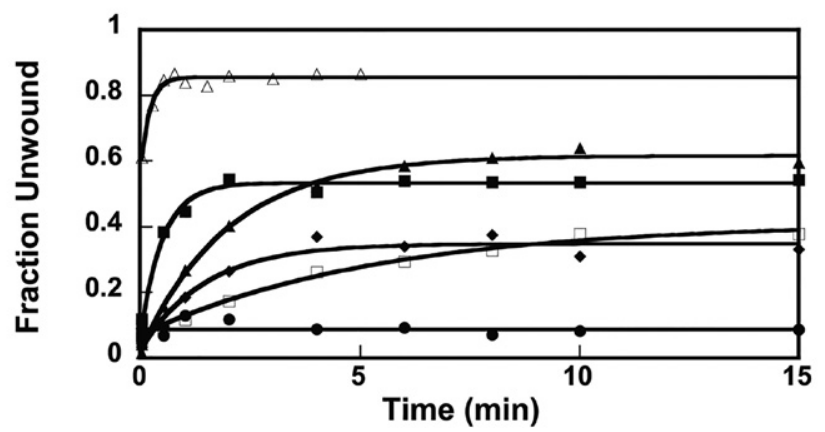

FIGURE 1. (A) Model molecules used to investigate the initiation site of DbpA protein helicase activity. (B) Helicase activity of DbpA in the presence of molecule A:X (filled triangle), A:Y (empty triangle), B:X (circle), C:X (diamond), D:X (empty square), and E:X (filled square). These are representative of the helicase experiments performed in the presence of $600 \mathrm{nM} \mathrm{DbpA}$ concentration. The average values and standard deviations for the observed rate constants of unwinding at 100, 300, and $600 \mathrm{nM}$ of DbpA are shown in Table 1.

The observation that molecule A:Y supports the helicase activity of DbpA, while the molecule B:X does not, indicates a role for the single-stranded region $3^{\prime}$ of a double-helix substrate on DbpA helicase activity. To shed light on the role of single-stranded region $3^{\prime}$ of the double-helix substrate and regions within the double-helix on the DbpA protein's catalytic activity, helicase experiments were performed in the presence of 32-nt-long constructs that had five residues at different positions from helix 92 changed to DNA. These are the molecules: C:X, D:X, and E:X in Figure 1A.

All the molecules with five RNA residues changed to DNA support the DbpA protein's helicase activity (Fig. 1B).
However, the fraction of RNA unwound in the presence of molecules $\mathrm{C}: \mathrm{X}$ and $\mathrm{D}: \mathrm{X}$ is smaller than in the presence of molecule A:X and E:X. In addition, the observed rate constant of DbpA helicase activity is different in the presence of different molecules. The fact that molecule E: $\mathrm{X}$ supports the DbpA unwinding suggests that the single-stranded region $3^{\prime}$ of the double-helix substrate, and $5^{\prime}$ of helix 92, is not required for the DbpA catalytic core to start its helicase activity. If this region were mechanistically important for the DbpA protein helicase activity, then molecule E:X, similar to molecule B:X, would not support double-helix unwinding.

To understand how the $2^{\prime}-\mathrm{OH}$ groups $5^{\prime}$ of helix 92 influence the amplitude and the observed rate constant of DbpA helicase reaction, we investigated the ability of $\mathrm{A}, \mathrm{B}, \mathrm{C}, \mathrm{D}$, and $\mathrm{E}$ constructs (Fig. 1A) to stimulate the ATPase activity of DbpA at a series of ATP and nucleic acid concentrations. The ability of the 32-nt-long constructs to activate the ATP hydrolysis activity of DbpA was investigated by the enzymatically coupled ATP/ $\mathrm{NADH}$ assay (Tsu and Uhlenbeck 1998; Elles and Uhlenbeck 2008). Figure 2A shows the dependence of the DbpA ATP hydrolysis rate at a series of ATP concentrations and in the presence of an excess of nucleic acid (2000 nM), while Figure 2B shows the rate of nucleic acid turnover at different 32-nt-long construct concentrations and in the presence of excess ATP $(5 \mathrm{mM})$. The MichaelisMenten equation was used to fit the data and the kinetic and thermodynamic parameters for those fits are shown in Table 2.

All constructs stimulate ATP hydrolysis; however, the maximum rate of ATP hydrolysis and the apparent affinity for ATP is considerably reduced in the presence of constructs $\mathrm{B}, \mathrm{C}$, and $\mathrm{D}$ when compared with the RNA constructs $\mathrm{A}$ and E (Table 2). DbpA binds the B, C, and D constructs with similar affinity to constructs $A$ and $E$, while in the presence of the $\mathrm{B}, \mathrm{C}$, and $\mathrm{D}$ constructs the turnover number of nucleic acid is reduced compared to constructs A and E (Table 2; Fig. 2).

The observation that in the presence of constructs B, C, and D the DbpA catalytic core does not hydrolyze ATP as efficiently and binds it with a lower affinity than in the presence 
TABLE 1. Helicase activity of DbpA interacting with various model molecules

\begin{tabular}{lccc}
\hline & \multicolumn{3}{c}{$k_{\text {obs }}\left(\mathrm{min}^{-1}\right)$} \\
\cline { 2 - 4 } Molecule & $100 \mathrm{nM} \mathrm{DbpA}$ & $300 \mathrm{nM}$ DbpA & $600 \mathrm{nM} \mathrm{DbpA}$ \\
\hline A:X & $0.43 \pm 0.16$ & $0.38 \pm 0.23$ & $0.39 \pm 0.15$ \\
B:X & $\sim 0$ & $\sim 0$ & $\sim 0$ \\
C:X & $0.66 \pm 0.11$ & $1.00 \pm 0.59$ & $1.00 \pm 0.66$ \\
D:X & $0.13 \pm 0.04$ & $0.15 \pm 0.02$ & $0.21 \pm 0.02$ \\
E:X & $1.37 \pm 0.69$ & $1.57 \pm 0.23$ & $2.16 \pm 0.42$ \\
A:Y & $3.35 \pm 2.08$ & $2.60 \pm 0.79$ & $4.16 \pm 1.11$ \\
F:Z & $0.15 \pm 0.02$ & $0.24 \pm 0.19$ & $0.30 \pm 0.10$ \\
G:Z & $0.14 \pm 0.03$ & $0.21 \pm 0.03$ & $0.29 \pm 0.10$ \\
\hline
\end{tabular}

The observed rate of unwinding was obtained by fitting the single exponential equation to the helicase assay as previously described (Diges and Uhlenbeck 2001). The values represent the means from at least two independent experiments and the errors are the deviation from the means. Molecule B:X is unable to support the DbpA helicase activity at $1000 \mathrm{nM}$ or $2000 \mathrm{nM}$ DbpA. The observed rate constant of unwinding at $1000 \mathrm{nM} \mathrm{DbpA}$ and in the presence of molecule $\mathrm{D}: \mathrm{X}$ is $0.16 \pm 0.08$, which is consistent with saturation of the helicase assay at $100 \mathrm{nM}$ DbpA for all the constructs employed in this study.

of constructs $\mathrm{A}$ and $\mathrm{E}$ implies that the lack of $2^{\prime}-\mathrm{OH}$ groups prevents the proper formation of the ATPase pocket. Combined with the observation that the extent of double-helix unwinding is reduced in molecules $\mathrm{B}: \mathrm{X}, \mathrm{C}: \mathrm{X}$, and $\mathrm{D}: \mathrm{X}$ when compared to molecules A:X and E:X, the ATPase data suggest that a fraction of DbpA molecules that contain the improperly formed ATPase pocket are stuck in an inactive intermediate conformation and unable to perform doublehelix unwinding.

For the DEAD-box family of proteins, the observed rate constant of double-helix unwinding is dependent on the unwinding catalytic efficiency of the enzyme, which is dependent on the rate of ATP hydrolysis, the stability of double helix, and the ability of the enzyme to perform annealing (Pyle 2008; Pan and Russell 2010; Henn et al. 2012; Putnam and Jankowsky 2013). Previous experiments have shown that the observed rate constant of DbpA-facilitated annealing is very similar to the observed rate constant of BSA-facilitated annealing; hence the DbpA protein does not seem to actively facilitate double-helix unwinding (O Uhlenbeck and C Diges, pers. comm.). In the case of molecules $B: X, A: X$, and $E: X$, the observed rate constant of helicase unwinding increases as the maximum rate of ATP hydrolysis increases, suggesting that the observed differences on the observed rate constant of unwinding in the presence of these molecules are a consequence of the differences on the DbpA catalytic core interaction with the bottom strand of the double-helix. If significant construct-dependent annealing was occurring, the maximum rate of ATP hydrolysis and RNA unwinding would not be expected to follow a similar trend.

Construct $\mathrm{C}$ stimulates the ATPase activity of DbpA with a much lower ATP and nucleic acid turnover than molecule A.
Surprisingly, although construct C stimulates the ATPase activity of DbpA less than construct A, molecule C:X supports the helicase activity of DbpA with a observed rate constant of unwinding similar to molecule A:X (Tables 1,2). The higher than expected observed rate constant of unwinding observed in the presence of construct C:X could be a consequence of the fact that the double-helix in molecule C:X contains three DNA residues. Therefore, the stability of the double-helix in molecule C:X is reduced in comparison to molecule A:X. Helix stability has been shown to influence the DEAD-box proteins' observed rate constants of unwinding (Chen et al. 2008).

Construct D stimulates the ATPase activity of DbpA similar to construct $\mathrm{C}$ as expected from the fact that both constructs contain five DNA bases $5^{\prime}$ of helix 92 . The number of DNA bases that molecules C:X and D:X contain in the double helix differ; molecule D:X contains five DNA bases, whereas molecule C:X contains only three. Due to the larger number of DNA bases on molecule D:X as opposed to C:X, the double helix in molecule $\mathrm{D}: \mathrm{X}$ is expected to be less stable than the double helix in molecule C:X. Based on double-helix stability and stimulation of DbpA ATPase activity, the observed rate constant of double-helix unwinding in the presence of construct D:X should be higher than the observed rate constant of double-helix unwinding in the presence of molecule C:X; however, the data on Figure 1 and Table 1 show the opposite trend.

The discrepancies between the stimulation of the ATPase activity, helix stability, and the observed rate constant of double-helix unwinding in the presence of molecule D:X could be a consequence of the low number of RNA bases on the double-helix region of this molecule. There are only four RNA residues that the DbpA catalytic core could interact within the double helix of molecule $\mathrm{D}: \mathrm{X}$, three at the $5^{\prime}$ end and one at the $3^{\prime}$ end. Thus, the loading of the DbpA catalytic core onto only a few regions of the double
A

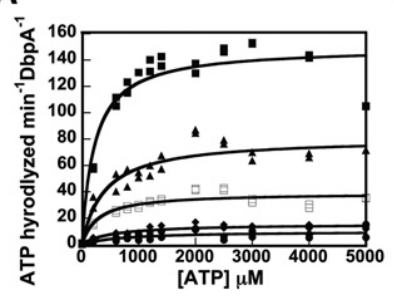

B

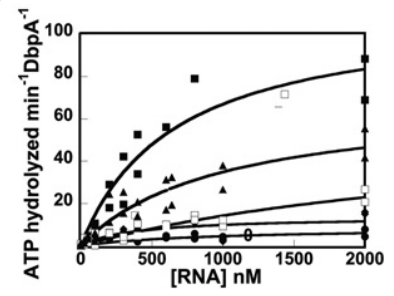

FIGURE 2. The role of $2^{\prime}-\mathrm{OH}$ moieties in DbpA ATPase activity. $(A)$ Activation of the ATPase activity of DbpA with a titration of ATP* Mg in the presence of $2000 \mathrm{nM}$ of A construct (filled triangle), B construct (circle), C construct (diamond), D construct (empty square), and E construct (filled square). (B) Activation of the ATPase activity of DbpA with titrations of the A construct (filled triangle), B construct (circle), C construct (diamond), D construct (empty square), and E construct (filled square) in the presence of $5 \mathrm{mM}$ ATP. The data shown represent one experiment. The average values and standard deviations for the kinetics and thermodynamic parameters obtained from fitting the Michaelis-Menten equation to similar data are shown in Table 2. 
Table 2. Kinetics and equilibrium parameters of DbpA interacting with 32-nt-long nucleic acid constructs

\begin{tabular}{lcccc}
\hline RNA & $\begin{array}{c}k_{\text {cat }}(\text { ATP })^{\mathrm{a}} \\
\left(\mathrm{min}^{-1}\right)\end{array}$ & $\begin{array}{c}K_{\mathrm{m}}(\mathrm{ATP})^{\mathrm{a}} \\
(\mu \mathrm{M})\end{array}$ & $\begin{array}{c}k_{\max }(\mathrm{RNA})^{\mathrm{b}} \\
\left(\mathrm{min}^{-1}\right)\end{array}$ & $\begin{array}{c}K_{\text {app }}(\mathrm{RNA})^{\mathrm{b}} \\
(\mathrm{nM})\end{array}$ \\
\hline $\mathrm{A}$ & $76 \pm 12$ & $337 \pm 72$ & $67 \pm 3$ & $1092 \pm 152$ \\
$\mathrm{~B}$ & $10 \pm 3$ & $743 \pm 105$ & $9 \pm 1$ & $987 \pm 64$ \\
$\mathrm{C}$ & $15 \pm 1$ & $648 \pm 115$ & $15 \pm 3$ & $605 \pm 291$ \\
$\mathrm{D}$ & $53 \pm 13$ & $368 \pm 170$ & $25 \pm 10$ & $1095 \pm 468$ \\
$\mathrm{E}$ & $127 \pm 20$ & $289 \pm 84$ & $179 \pm 72$ & $946 \pm 460$ \\
\hline
\end{tabular}

${ }^{\mathrm{a}}$ The turnover numbers for ATP hydrolysis and the ATP MichaelisMenten constant were obtained by fitting the Michaelis-Menten equation to the dependence of ATP hydrolysis rate on the ATP concentration data. The values represent the means from three independent experiments and the errors are deviations from the means.

'The nucleic acid turnover number and the DbpA protein's apparent binding affinity for the nucleic acid were calculated for the fit of Michaelis-Menten equation. The values represent the average of two independent experiments and the errors are deviations from these averages

helix would produce unwinding. For every encounter of the DbpA catalytic core with the substrate helix, there is a smaller chance for the helix to unwind in molecule D:X than in molecule C:X. In addition, while the DbpA catalytic core contacts with a DNA-RNA region may produce unwinding, the unwinding process could be slower because of the lack of proper interactions between the catalytic core and the DNA residues. The contacts between the catalytic core and DNA residues would be more numerous in molecule $\mathrm{D}: \mathrm{X}$ than in $\mathrm{C}: \mathrm{X}$. This could be another plausible explanation for the low observed rate constant of double-helix unwinding observed in the presence of molecule D:X when compared to molecule $\mathrm{C}: \mathrm{X}$. A decrease in the observed rate constant of unwinding with an increase in the number of double-helix substrate DNA bases has also been observed for Ded1p and Mss116p DEAD-box helicases (Yang and Jankowsky 2005).

Although it is possible that the smaller than expected observed rate constant of unwinding observed in the presence of molecule D:X is a consequence of DbpA annealing activity, taking into account that no annealing activity was previously observed for the DbpA protein and that the observed rate constant of unwinding for molecules A:X, B:X, C:X, and E: $\mathrm{X}$ follows the same trend as the ATPase activity and the helix stability, we believe that the slow observed rate constant of unwinding in the presence of construct $\mathrm{D}: \mathrm{X}$ is a consequence of the lack of $2^{\prime}-\mathrm{OH}$ group on the bottom strand of this construct. Detailed experiments investigating the activation of DbpA annealing by molecule D:X, although interesting, are outside the scope of this study.

The observation that construct E stimulates DbpA's ATP and nucleic acid turnover better than construct A (Table 2; Fig. 2), and molecule E:X stimulates a higher observed rate constant of DbpA unwinding than molecule A:X (Table 1; Fig. 1) implies that the DNA segment $5^{\prime}$ of helix 92 is interacting with the DbpA C-terminal domain differently than the
RNA segment in the same region. As the apparent binding affinity of the DbpA protein for the E construct and the A construct are similar (Table 2), the DNA segment does not seem to increase the affinity of the protein for construct E; however, the DNA segment may affect the way the 10 RNA residues $5^{\prime}$ of the DNA region are presented to the DbpA catalytic core. More importantly, these experiments suggest that the DNA region in construct $\mathrm{E}$, which is the region $3^{\prime}$ of the double-helix substrate in the molecule $\mathrm{E}: \mathrm{X}$, is not interacting with the catalytic core of DbpA. If the DNA region were interacting with the DbpA catalytic core, the ATPase activity of DbpA would have decreased. Thus, the DbpA catalytic core could not start its helicase activity at the $3^{\prime}$ end of the double helix. The only mechanism that the DbpA catalytic core could employ to unwind the double-helix substrate on the E:X molecule is by directly loading on this helix.

One explanation of why the previous studies suggested that the region between helix 92 and the double-helix substrate was required to be single stranded in order for an RNA molecule to support the helicase activity of DbpA (Diges and Uhlenbeck 2001, 2005) is that the single-stranded region could be required for the tight interaction of the DbpA Cterminal domain with the RNA molecule. Modeling of the interaction of an RNA molecule, containing helix 92 and five residues $5^{\prime}$ of this helix, with the YxiN C-terminal domain shows that the five-residue single-stranded region wraps around the protein (Hardin et al. 2010). A doublestranded RNA region, which has a higher persistence length than the single-stranded region, probably could not wrap around the protein (Kebbekus et al. 1995; Abels et al. 2005; Bizarro et al. 2012; Chen et al. 2012). In addition, the modeling study shows that the single-stranded region $5^{\prime}$ of helix 92 interacts with the C-terminal of YxiN via nonspecific charge-charge interactions (Hardin et al. 2010), and a single-stranded DNA segment, such as the one in the molecule $\mathrm{E}: \mathrm{X}$, could satisfy these interactions.

Comparison of the DbpA protein's helicase activity in the presence of molecules $\mathrm{A}: \mathrm{Y}, \mathrm{B}: \mathrm{X}$, and $\mathrm{E}: \mathrm{X}$ suggests that the top and bottom strands of the double helix interact differently with the DbpA protein (Fig. 1). One possibility is that the top RNA strand of the double-helix on the molecule B:X, could be interacting with the C-terminal binding domain of $\mathrm{DbpA}$, and the 32-nt-long strand, which lacks the $2^{\prime}-\mathrm{OH}$ groups $5^{\prime}$ of hairpin 92 , is the only strand accessible to the DbpA catalytic core. To test this hypothesis, a new construct was designed in which all the residues $5^{\prime}$ of helix 92 are DNA, but the single-stranded region is $23 \mathrm{nt}$ longer than the singlestranded region in molecule B (Fig. 3A molecule F:Z). The longer construct places the double-helix substrate farther in space from helix and hairpin 92 where the C-terminal domain of DbpA is anchored. As a consequence, the C-terminal domain of DbpA could not interact with the top strand of the double helix. The data in Figure $3 \mathrm{~B}$ show that the new longer construct supports the helicase activity of the DbpA protein. Thus, the DbpA protein's inability to unzip the 


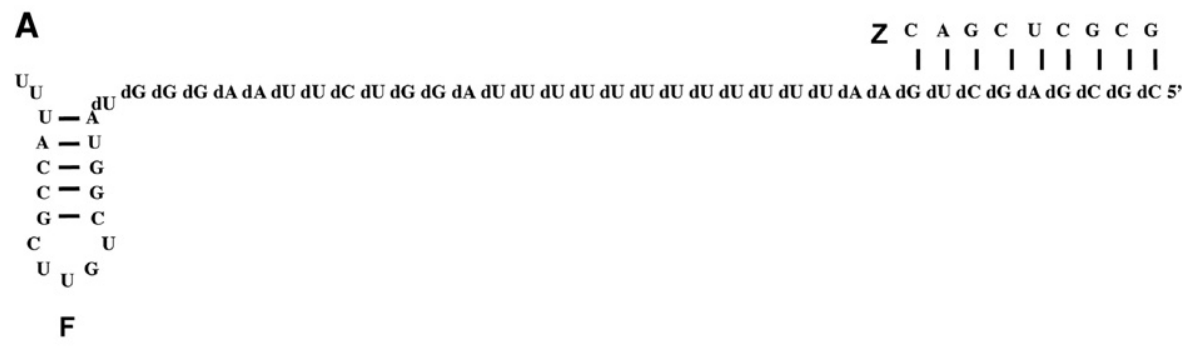

B

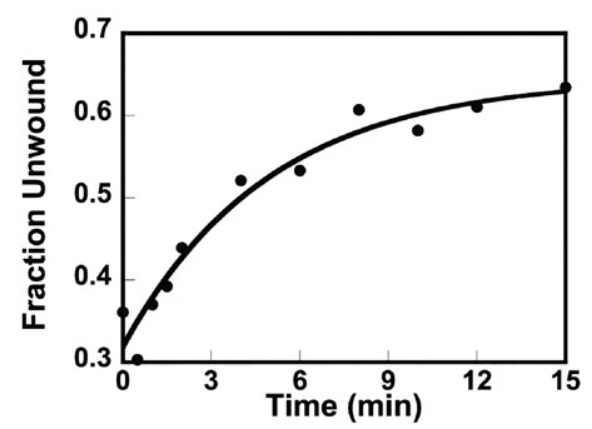

FIGURE 3. Effect of physical distance between the double-helix substrate and helix 92 on the DbpA protein helicase activity. $(A)$ The model RNA-DNA chimera used for this experiment. $(B)$ Helicase activity of the DbpA protein in the presence of longer RNA-DNA chimera. These data represent one experiment performed at $600 \mathrm{nM}$ DbpA. The observed rate constants of unwinding average values obtained by multiple independent experiments and various DbpA concentrations are shown in Table 1 .

RNA strand annealed to molecule B:X is a consequence of the top RNA strand of the double helix interacting with the DbpA RNA-binding domain. Consequently, this strand is inaccessible to interact with the DbpA catalytic core, while the bottom DNA strand, which is accessible to interact with the DbpA catalytic core, lacks the 2 '-OH groups required to support the DbpA helicase activity. The experiments performed with molecule F:Z (Fig. 3) further imply that the singlestranded region $3^{\prime}$ of the double helix is not the DbpA protein's entry site. All the residues $3^{\prime}$ of the double-helix substrate and on the bottom strand of the double-helix substrate are DNA in this construct.

\section{RNA-polyethylene glycol chimera supports DbpA helicase activity}

To further investigate the possibility that the DbpA protein employs the direct loading mechanism, we designed a model molecule in which three ethylene glycol residues are placed $3^{\prime}$ of double-helix substrate and two residues removed from it (Fig. 4A). Similar experiments with polyethylene glycol nucleic acid chimeras have previously been performed with NPH-II RNA helicase from the hepatitis C virus (Kawaoka et al. 2004) and the E. coli Rep Helicase (Amaratunga and Lohman 1993).

The ability of the RNA-polyethylene glycol chimera to support the DbpA protein's helicase activity was measured by native gel assay. Figure 4 shows the dependence of an RNA fraction unwound versus the reaction time. These data demonstrate that the RNA-polyethylene glycol chimera supports the DbpA protein's unwinding activity.

The fact that an RNA-polyethylene glycol chimera supports the helicase activity of DbpA, although polyethylene glycol is not a physiological polymer and there are no chemical groups in polyethylene glycol that the catalytic core of DbpA could recognize, further demonstrates that the four singlestranded residues $3^{\prime}$ of the double-helix substrate are not required to support the DbpA protein's helicase activity, and that the DbpA protein, similar to other members of the DEAD-box family, unwinds the double-helix substrates by directly loading on them.

\section{DISCUSSION}

The DbpA protein helicase and ATPase data collected in the presence of both the DNA-RNA and RNApolyethylene glycol chimeras shed light on the RNA groups required to support the DbpA helicase activity. These data demonstrate that the residues outside the double-helix region are mechanistically unimportant for the DbpA catalytic activity and the single-stranded region $3^{\prime}$ of the double-helix substrate is not the DbpA protein's helicase action entry site. Similar to other members of the DEAD-box family of enzymes, the DbpA catalytic core employs the direct loading mechanism to unwind its doublehelix substrate.

Within the nine-residue double-helix region, when the top strand is interacting with the DbpA RNA-binding domain, as few as four RNA residues on the bottom strand are sufficient for the support of DbpA helicase activity. These results agree with previous experimental data performed with Ded1p and Mss116p. In those experiments, when one strand of a doublehelix was DNA, not all the residues on the other strands were required to be RNA in order for the helix to support the helicase activity of Ded1p and Mss116p (Yang et al. 2007).

If the DbpA protein employs the direct loading mechanism to unwind its substrate, then why was this mechanism not observed in the previous study (Diges and Uhlenbeck 2005)? Instead, it was suggested that the DbpA protein starts its action at a single-stranded region $3^{\prime}$ end of the doublehelix substrate (Diges and Uhlenbeck 2001, 2005). The data shown here combined with the crystal structure and previous biochemical data suggest that the DbpA RNA-binding domain contacts different RNA regions in different RNA model substrates (Tsu et al. 2001; Hardin et al. 2010). The 
A

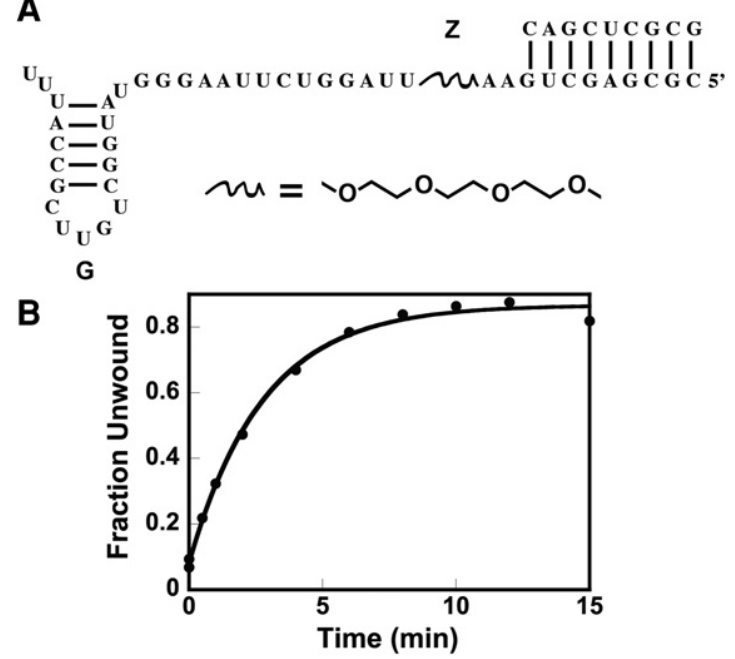

FIGURE 4. Effect of polyethylene glycol linker on the function properties of DbpA. (A) The RNA-polyethylene glycol chimera construct used for these experiments. The nine-atom ethylene glycol linker spans three RNA bases. (B) Helicase activity of DbpA in the presence of RNA-polyethylene glycol chimera. These data represent one experiment performed at $600 \mathrm{nM}$ DbpA. The observed rate constants of unwinding average values obtained by multiple independent experiments and various DbpA concentrations are shown in Table 1.

interaction of the DbpA RNA-binding domain with an RNA molecule modulates both the affinity of the DbpA protein for that RNA molecule and the helix substrates accessible to the DbpA catalytic core for unwinding. Therefore, it must be the asymmetric interaction of the DbpA RNA-binding domain with the model RNA molecules used in the previous study that gave the appearance of a symmetric interaction of the DbpA catalytic core with its double-helix substrate (Diges and Uhlenbeck 2005).

The fact that the DbpA protein employs the direct loading mechanism to unwind double-helix substrates has important implications for the role of $\mathrm{DbpA}$ during ribosome assembly in vivo. It suggests that during the ribosome assembly process, DbpA could unwind any substrates within the catalytic core's reach by directly loading on them. The double-helix substrates could be far in sequence from helix 92 and separated from helix 92 by space or other macromolecules. Exceptions to the substrates that DbpA unwinds are the double-helix regions that directly interact with its C-terminal domain.

The precise RNA regions in addition to helix and hairpin 92 that the C-terminal and the catalytic core contacts during the in vivo large subunit ribosome assembly remain unknown. Determination of these regions may shed light on the role of the C-terminal of DbpA on in vivo large ribosome assembly. For instance, it is possible that the C-terminal domain interaction with a correctly folded RNA double helix during assembly of the large ribosomal subunit prevents the DbpA catalytic core from unwinding that helix. Hence, the C-terminal domain of DbpA, besides its known role of deliv- ering the DbpA protein to its site of action on the peptide bond formation site, could also have the role of preventing the DbpA catalytic core access to correctly formed double helices, which do not require unwinding.

\section{MATERIALS AND METHODS}

\section{RNA molecules and enzymes}

All RNA and DNA constructs, RNA-DNA, and RNA-polyethylene glycol chimeras were purchased HPLC purified from IDT. Poly(A) was purchased from Sigma-Aldrich. Pyruvate kinase/lactate dehydrogenase enzyme mixture was purchased from Sigma-Aldrich as a buffered aqueous glycerol solution.

\section{Protein purification}

DbpA was purified as previously described (Elles and Uhlenbeck 2008 ) with the sole exception that $\mathrm{KCl}$ was used in all purification and dialysis buffers instead of $\mathrm{NaCl}$.

\section{ATPase activity}

The rate of ATP hydrolysis and nucleic acid turnover were measured as previously described (Tsu and Uhlenbeck 1998). The $K_{\mathrm{M}}$ (the Michaelis constant) and $k_{\text {cat }}$ (the turnover rate) were obtained from fitting the Michaelis-Menten equation to the data shown in Figure 2A,B.

\section{Helicase assay}

The annealing and the helicase assay conditions used were similar to those previously described with a few modifications (Diges and Uhlenbeck 2005). The reaction was applied to a $20 \%$ acrylamide native gel (29:1 acrylamide to bis-acrylamide ratio) at $4^{\circ} \mathrm{C} .5 \mathrm{mM}$ $\mathrm{MgCl}_{2}$ was present in both the gel and the gel running buffer. The fraction of unwound RNA or DNA was calculated by the ratio of the counts on the single-stranded band over the sum of the counts on the annealed band plus the single-stranded band. The data were fit to the equation:

$$
f_{u}=f_{u}(0)+A(1-\exp (-k t)),
$$

where $f_{\mathrm{u}}$ is the fraction of the double helix unwound, $f_{\mathrm{u}}(0)$ is the fraction of the double helix unwound before the addition of ATP, $A$ is the amplitude of unwinding transition, and $k$ is the observed rate constant of unwinding (Diges and Uhlenbeck 2001; Chen et al. 2008; Elles and Uhlenbeck 2008).

Spontaneous unwinding was observed for molecules A:Y an F:Z. For these molecules the observed rate constant of unwinding is calculated from the differences in the observed rate constants of unwinding in the presence and absence of ATP (Chen et al. 2008).

\section{ACKNOWLEDGMENTS}

A part of these data was collected in O. Uhlenbeck's laboratory at Northwestern University. E.K. thanks O. Uhlenbeck and M. Saks for many helpful discussions. This work was supported by 
National Institutes of Health (5R21CA175625-02 to E.K.) and University of Central Florida (in-house grant to E.K.).

Received June 21, 2015; accepted December 2, 2015.

\section{REFERENCES}

Abels JA, Moreno-Herrero F, van der Heijden T, Dekker C, Dekker NH. 2005. Single-molecule measurements of the persistence length of double-stranded RNA. Biophys J 88: 2737-2744.

Amaratunga M, Lohman TM. 1993. Escherichia coli rep helicase unwinds DNA by an active mechanism. Biochemistry 32: 6815-6820.

Andersen CB, Ballut L, Johansen JS, Chamieh $\mathrm{H}$, Nielsen $\mathrm{KH}$, Oliveira CL, Pedersen JS, Seraphin B, Le Hir H, Andersen GR. 2006. Structure of the exon junction core complex with a trapped DEAD-box ATPase bound to RNA. Science 313: 1968-1972.

Aregger R, Klostermeier D. 2009. The DEAD box helicase YxiN maintains a closed conformation during ATP hydrolysis. Biochemistry 48: 10679-10681.

Bizarro CV, Alemany A, Ritort F. 2012. Non-specific binding of $\mathrm{Na}^{+}$and $\mathrm{Mg}^{2+}$ to RNA determined by force spectroscopy methods. Nucleic Acids Res 40: 6922-6935.

Bono F, Ebert J, Lorentzen E, Conti E. 2006. The crystal structure of the exon junction complex reveals how it maintains a stable grip on mRNA. Cell 126: 713-725.

Caruthers JM, McKay DB. 2002. Helicase structure and mechanism. Curr Opin Struct Biol 12: 123-133.

Chen Y, Potratz JP, Tijerina P, Del Campo M, Lambowitz AM, Russell R. 2008. DEAD-box proteins can completely separate an RNA duplex using a single ATP. Proc Natl Acad Sci 105: 20203-20208.

Chen H, Meisburger SP, Pabit SA, Sutton JL, Webb WW, Pollack L. 2012. Ionic strength-dependent persistence lengths of single-stranded RNA and DNA. Proc Natl Acad Sci 109: 799-804.

Cordin O, Banroques J, Tanner NK, Linder P. 2006. The DEAD-box protein family of RNA helicases. Gene 367 : 17-37.

Del Campo M, Lambowitz AM. 2009. Structure of the Yeast DEAD box protein Mss116p reveals two wedges that crimp RNA. Mol Cell 35: 598-609.

Diges CM, Uhlenbeck OC. 2001. Escherichia coli DbpA is an RNA helicase that requires hairpin 92 of $23 \mathrm{~S}$ rRNA. EMBO J 20: 5503-5512.

Diges CM, Uhlenbeck OC. 2005. Escherichia coli DbpA is a $3^{\prime} \rightarrow 5^{\prime}$ RNA helicase. Biochemistry 44: 7903-7911.

Elles LM, Uhlenbeck OC. 2008. Mutation of the arginine finger in the active site of Escherichia coli DbpA abolishes ATPase and helicase activity and confers a dominant slow growth phenotype. Nucleic Acids Res 36: 41-50.

Fairman-Williams ME, Guenther UP, Jankowsky E. 2010. SF1 and SF2 helicases: family matters. Curr Opin Struct Biol 20: 313-324.

Fuller-Pace FV, Nicol SM, Reid AD, Lane DP. 1993. DbpA: a DEAD box protein specifically activated by $23 \mathrm{~s}$ rRNA. EMBO $J$ 12: 3619-3626.

Hardin JW, Hu YX, McKay DB. 2010. Structure of the RNA binding domain of a DEAD-box helicase bound to its ribosomal RNA target reveals a novel mode of recognition by an RNA recognition motif. $J$ Mol Biol 402: 412-427.

Henn A, Cao W, Hackney DD, De La Cruz EM. 2008. The ATPase cycle mechanism of the DEAD-box rRNA helicase, DbpA. J Mol Biol 377: 193-205.

Henn A, Bradley MJ, De La Cruz EM. 2012. ATP utilization and RNA conformational rearrangement by DEAD-box proteins. Annu Rev Biophys 41: 247-267.

Karginov FV, Caruthers JM, Hu Y, McKay DB, Uhlenbeck OC. 2005. YxiN is a modular protein combining a $\operatorname{DEx}(\mathrm{D} / \mathrm{H})$ core and a specific RNA-binding domain. J Biol Chem 280: 35499-35505.

Kawaoka J, Jankowsky E, Pyle AM. 2004. Backbone tracking by the SF2 helicase NPH-II. Nat Struct Mol Biol 11: 526-530.

Kebbekus P, Draper DE, Hagerman P. 1995. Persistence length of RNA. Biochemistry 34: 4354-4357.

Kossen K, Uhlenbeck OC. 1999. Cloning and biochemical characterization of Bacillus subtilis YxiN, a DEAD protein specifically activated by $23 \mathrm{~S}$ rRNA: delineation of a novel sub-family of bacterial DEAD proteins. Nucleic Acids Res 27: 3811-3820.

Linder P, Jankowsky E. 2011. From unwinding to clamping - the DEAD box RNA helicase family. Nat Rev Mol Cell Biol 12: 505-516.

Pan C, Russell R. 2010. Roles of DEAD-box proteins in RNA and RNP folding. RNA Biol 7: 28-37.

Putnam AA, Jankowsky E. 2013. DEAD-box helicases as integrators of RNA, nucleotide and protein binding. Biochim Biophys Acta 1829: 884-893.

Pyle AM. 2008. Translocation and unwinding mechanisms of RNA and DNA helicases. Annu Rev Biophys 37: 317-336.

Sengoku T, Nureki O, Nakamura A, Kobayashi S, Yokoyama S. 2006. Structural basis for RNA unwinding by the DEAD-box protein Drosophila Vasa. Cell 125: 287-300.

Sharpe Elles LM, Sykes MT, Williamson JR, Uhlenbeck OC. 2009. A dominant negative mutant of the E. coli RNA helicase DbpA blocks assembly of the $50 \mathrm{~S}$ ribosomal subunit. Nucleic Acids Res 37: 6503-6514.

Tanner NK, Linder P. 2001. DExD/H box RNA helicases: from generic motors to specific dissociation functions. Mol Cell 8: 251-262.

Theissen B, Karow AR, Kohler J, Gubaev A, Klostermeier D. 2008. Cooperative binding of ATP and RNA induces a closed conformation in a DEAD box RNA helicase. Proc Natl Acad Sci 105: 548-553.

Tsu CA, Uhlenbeck OC. 1998. Kinetic analysis of the RNA-dependent adenosinetriphosphatase activity of DbpA, an Escherichia coli DEAD protein specific for $23 \mathrm{~S}$ ribosomal RNA. Biochemistry 37: 16989-16996.

Tsu CA, Kossen K, Uhlenbeck OC. 2001. The Escherichia coli DEAD protein DbpA recognizes a small RNA hairpin in 23S rRNA. RNA 7: 702-709.

Wang S, Hu Y, Overgaard MT, Karginov FV, Uhlenbeck OC, McKay DB. 2006. The domain of the Bacillus subtilis DEAD-box helicase YxiN that is responsible for specific binding of $23 \mathrm{~S}$ rRNA has an RNA recognition motif fold. RNA 12: 959-967.

Yang Q, Jankowsky E. 2005. ATP- and ADP-dependent modulation of RNA unwinding and strand annealing activities by the DEAD-box protein DED1. Biochemistry 44: 13591-13601.

Yang Q, Del Campo M, Lambowitz AM, Jankowsky E. 2007. DEAD-box proteins unwind duplexes by local strand separation. Mol Cell 28: 253-263. 

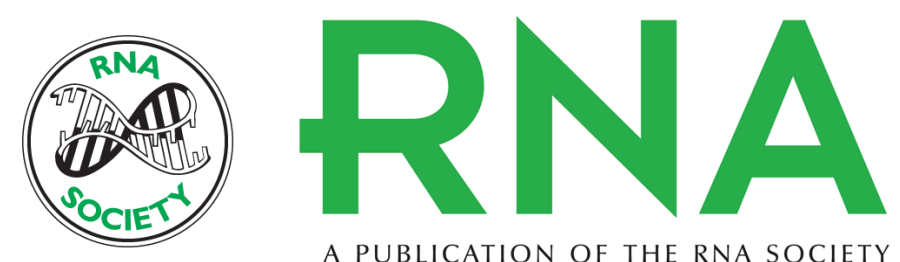

A PUBLICATION OF THE RNA SOCIETY

\title{
The DbpA catalytic core unwinds double-helix substrates by directly loading on them
}

Jared J. Childs, Riley C. Gentry, Anthony F.T. Moore, et al.

RNA 2016 22: 408-415 originally published online January 11, 2016

Access the most recent version at doi:10.1261/rna.052928.115

\begin{abstract}
References This article cites 36 articles, 8 of which can be accessed free at: http://rnajournal.cshlp.org/content/22/3/408.full.html\#ref-list-1

Creative This article is distributed exclusively by the RNA Society for the first 12 months after the Commons full-issue publication date (see http://rnajournal.cshlp.org/site/misc/terms.xhtml). After 12 License months, it is available under a Creative Commons License (Attribution-NonCommercial 4.0 International), as described at http://creativecommons.org/licenses/by-nc/4.0/.
\end{abstract}

Email Alerting Receive free email alerts when new articles cite this article - sign up in the box at the Service top right corner of the article or click here.

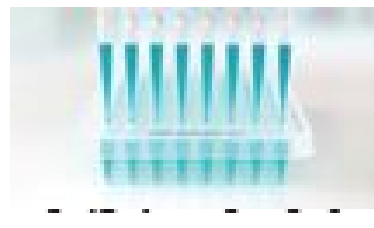

\section{Providing Precise Solutions for} your research.

To subscribe to RNA go to:

http://rnajournal.cshlp.org/subscriptions 